\title{
Revisão
}

\section{Apoio matricial como ferramenta de articulação entre a saúde mental e a atenção primária à saúde}

\author{
Support matrix as a tool for coordination \\ between mental health and primary health care
}

\author{
Dana Karine de Sousa Machado ${ }^{1}$, Marcio Wagner Camatta²
}

\begin{abstract}
Resumo
O objetivo deste trabalho foi caracterizar a utilização do apoio matricial como instrumento de articulação entre a Saúde Mental e a Atenção Primária, identificando suas fragilidades e potencialidades. Trata-se de uma Revisão Integrativa, na qual se utilizaram artigos indexados na base PubMed, na NLM e na BVS, a qual dá acesso às bases de dados LILACS, MEDLINE e IBECS, no período de 1999 a 2013, em português, inglês e espanhol. A amostra incluiu 21 artigos, e sua análise permitiu a identificação de duas categorias: 1) aspectos que dificultam o matriciamento; 2) aspectos potencializadores do apoio matricial. Os resultados encontrados foram: $24 \%$ dos textos traziam apenas aspectos positivos da implementação do matriciamento; 28,5\% mostravam apenas resultados que impediam sua concretização; e 47,5\% dos artigos abordavam ambos os aspectos. As publicações evidenciaram que, embora existam aspectos que dificultam a sua implantação, o matriciamento, quando bem utilizado, mostra-se como uma importante ferramenta de interlocução entre a Saúde Mental e a atenção primária.

Palavras-chave: saúde mental; serviços de saúde mental; atenção primária à saúde.
\end{abstract}

\begin{abstract}
The aim of this study was to characterize the matrix support implementation as a coordination instrument between Mental Health and primary care, identifying its weaknesses and strengths. It is an Integrative Review, in which we selected some articles indexed in PubMed, besides NLM, and VHL, which provides the access to LILACS, MEDLINE and IBECS databases, from 1999 to 2013, in Portuguese, English and Spanish. The sample included 21 articles and the analysis allowed the identification of two categories: 1) aspects that hinder support matrix, and 2) aspects that potentiate the matrix support. The results brought out that: $24 \%$ of texts showed only positive aspects of the matrix support implementation; $28.5 \%$ showed only the results that hinder the execution; and $47.5 \%$ of the articles addressed both aspects. The publications revealed that, although there are aspects that preclude its achievement, matrix support, when properly used, is an important tool for the dialogue between Mental Health and primary care.
\end{abstract}

Keywords: mental health; mental health services; primary health care.

Trabalho realizado no Curso de Pós-Graduação em Saúde da Família do Instituto de Educação e Pesquisa (IEP) do Hospital Moinhos de Vento - Porto Alegre (RS), Brasil.

${ }^{1}$ Enfermeira pela Universidade Federal do Rio Grande do Sul (UFRGS); Pós-Graduanda em Saúde da Família pelo IEP do Hospital Moinhos de Vento - Porto Alegre (RS), Brasil.

2Enfermeiro pela Universidade Federal do Espírito Santo (UFES); Doutor em Enfermagem pela UFRGS; Professor da Universidade Federal de Ciências da Saúde de Porto Alegre (UFCSPA) e do IEP do Hospital Moinhos de Vento - Porto Alegre (RS), Brasil.

Endereço para correspondência: Dana Karine de Sousa Machado - Rua Guadalajara, 32 - CEP: 91215-290 - Porto Alegre (RS), Brasil - E-mail: dana.sousa@gmail.com

Fonte de financiamento: nenhuma.

Conflito de interesse: nada a declarar. 


\section{INTRODUÇÃO}

A Atenção Primária à Saúde (APS) caracteriza-se por um conjunto de ações, no âmbito individual e coletivo, com o objetivo de desenvolver uma atenção integral que impacte na situação de saúde e autonomia das pessoas e nos determinantes e condicionantes de saúde das coletividades, tendo na Saúde da Família sua estratégia prioritária para expansão e consolidaçãoํ․ A Estratégia Saúde da Família (ESF) surge, portanto, para reorganizar a Atenção Primária no país e atua com equipes multiprofissionais responsáveis pelo cuidado integral, contínuo e resolutivo de um número definido de famílias e com um território adscrito ${ }^{2,3}$.

Criado com o intuito de dar suporte a essas equipes, o apoio matricial ou matriciamento pode ser entendido como a construção de momentos relacionais, em que se estabelecem trocas de saberes entre os profissionais. Apoiando os diferentes serviços de atenção envolvidos no cuidado dos usuários, como, por exemplo, uma Unidade Básica de Saúde (UBS) e um Centro de Atenção Psicossocial (CAPS) ${ }^{4}$, o matriciamento desmistifica a errônea ideia de saúde mental como "cuidado restrito aos loucos" e apoio matricial como "encaminhamento ao especialista".

Nesse cenário, percebe-se, no dia a dia, o quanto a interlocução propiciada pelo apoio matricial serve como facilitador na resolução das situações de saúde mental advindas da atenção primária, uma vez que muitos usuários não precisam chegar ao espaço de serviços especializados como os Centros de Atenção Psicossocial (CAPS) 5 . A grande maioria percorre diversos serviços de saúde e não veem atendidas suas necessidades, no que tange à saúde mental, já que os CAPS têm por prerrogativa atender e tratar pacientes com transtornos mentais graves e persistentes, ficando as demais situações, tais como depressão leve e moderada, ansiedade e esquizofrenia estáveis, a cargo da atenção primária ${ }^{1}$. Entretanto, frequentemente, os profissionais das equipes da atenção primária não se sentem preparados para lidar com essas situações ${ }^{6,7}$.

Além disso, a fragilidade das redes de cuidados estabelecidas, por vezes, não propiciam momentos de interlocução entre os serviços de maneira que atenda às necessidades particulares e demandas dos seus usuários ${ }^{8}$. Quem acaba sofrendo por essa condição sensível da rede são os usuários e suas famílias, que se beneficiariam com essa forma de cuidado colaborativo entre a atenção primária e a saúde mental.

Procurando contribuir e somar esforços para a excelência da assistência à saúde mental da população, a seguinte questão de pesquisa é proposta: Que fatores têm sido identificados como facilitadores e como dificultadores da interlocução entre Atenção Primária à Saúde e Saúde Mental, propiciada pelo matriciamento?
Este estudo justifica-se pela percepção de que os profissionais de saúde, tanto da atenção primária, quanto da saúde mental, possam acessar, apesar de não ser um serviço recente, pouca informação a respeito do tema matriciamento, deixando, assim, de conhecê-lo, estruturá-lo e usá-lo em sua prática. Dessa forma, usuários que poderiam ter suas demandas atendidas pela Estratégia de Saúde da Família acabam sendo, muitas vezes, encaminhados a um especialista, correndo o risco de não serem acolhidos em um espaço adequado, ou, o que é pior, não tendo suas necessidades atendidas.

O objetivo deste estudo foi caracterizar a utilização do apoio matricial como instrumento de articulação entre a Atenção Primária e a Saúde Mental. Para tanto, buscou-se identificar as fragilidades e potencialidades do apoio matricial na rede de atenção em saúde mental.

\section{REVISÃO DE LITERATURA}

\section{Atenção Primária, Saúde Mental e matriciamento}

As décadas de 1970 e 1980, no Brasil, foram de extrema importância para a saúde, uma vez que a intensa movimentação social que ocorreu nessa época, buscando-se a redemocratização do país e a melhoria das condições de vida da população, impulsionou uma fase de mobilização e discussão centrada na saúde. A partir das reivindicações sociais, iniciaram-se os processos da Reforma Sanitária e da Luta Antimanicomial, promulgou-se a nova Constituição Brasileira e estimulou-se a reestruturação da Atenção Básica.

A partir da mobilização da sociedade, potencializada, principalmente, por estudantes universitários, profissionais da saúde e cientistas sociais, ocorre, em 1986, um dos marcos na história da nossa Saúde Pública, a VIII Conferência Nacional de Saúde, que reuniu cerca de 4 mil pessoas em Brasília, a fim de discutir a Reforma Sanitária a ser implantada no país. Uma importante realização da VIII Conferência foi a inserção da saúde como direito de cidadania e dever do Estado, no texto da Constituição Brasileira de 1988, qualificando e dando força jurídica de relevância pública às ações e serviços de saúde. Dessa forma, a Constituição rompeu com o padrão anterior de política social, marcado pela exclusão de milhões de brasileiros do usufruto público de bens de consumo coletivo².

Concomitantemente a esse processo, ocorre o movimento da Luta Antimanicomial, que se deu devido a denúncias, por profissionais e familiares, dos abusos e maus-tratos sofridos pelos usuários da saúde mental, sendo fortemente influenciado por Franco Basaglia, psiquiatra italiano, que propunha a crítica radical ao manicômio e a desinstitucionalização como prática a ser seguida. Assim, eclode o movimento da Reforma Psiquiátrica, processo político e social complexo, que passa 
a ser compreendido como um conjunto de transformações de práticas, saberes, valores culturais e sociais, que propõe, além do resgate da autonomia e dos direitos dos pacientes, a substituição do modelo asilar e manicomial por uma rede de serviços de base territorial e comunitária ${ }^{9}$.

Paralelamente a esse contexto de lutas e buscas pela humanização do cuidado, ocorre, na cidade do Rio de Janeiro, a I Conferência Nacional de Saúde Mental, em 1987. No mesmo ano, surge o primeiro CAPS em São Paulo, e o início de um processo de intervenção, em 1989, da Secretaria Municipal de Saúde de Santos (SP) em um hospital psiquiátrico, a Casa de Saúde Anchieta, local de maus-tratos e mortes de pacientes 9 . Os CAPS são dispositivos de atenção à saúde direcionados a pessoas com transtornos mentais graves e persistentes, com o objetivo de organizar a rede substitutiva aos hospitais psiquiátricos nos municípios em que estão inseridos ${ }^{10}$.

Constituem um serviço comunitário e aberto, que oferece atendimentos diários, em um ambiente acolhedor, com equipe multiprofissional e população adscrita. Além disso, os CAPS têm como funções, entre outras, promover a inserção social das pessoas com transtornos mentais por meio de ações intersetoriais; regular a porta de entrada da rede de assistência em saúde mental na sua área de atuação; dar suporte a atenção à saúde mental na rede básica; e articular estrategicamente a rede e a política de saúde mental em seu território ${ }^{10}$.

Em 1988, com a aprovação da nova Constituição Brasileira, ocorre a instituição do Sistema Único de Saúde (SUS) como modelo de cuidado, a fim de que a população tenha acesso a um atendimento público de qualidade, resolutivo e gratuito. Já em 1989, o deputado Paulo Delgado dá entrada, no Congresso Nacional, a um projeto de lei que propõe a regulamentação dos direitos da pessoa com transtornos mentais e a extinção progressiva dos manicômios no país, mas que foi promulgado apenas em 2001 - a lei federal no 10. $216^{9}$.

Em 1994, surge o Programa de Saúde da Família, inicialmente com o objetivo de expandir a cobertura de saúde a cidades do interior dos municípios brasileiros, passando à estratégia em 1998. A Estratégia de Saúde da Família desenvolve seu trabalho por meio da atenção integral, humanizada, de alta resolutividade, com acessibilidade, acolhimento, vínculo, responsabilização pelo cuidado continuado das pessoas vinculadas a seu território, contando com uma equipe multiprofissional para atender às demandas de saúde da população $0^{2,3}$. Em 2012, já havia 32.865 equipes implantadas, com uma cobertura populacional de $54,2 \%$ da população brasileira, correspondendo a cerca de 104 milhões de pessoas ${ }^{11}$.

Essa nova forma de trabalho das equipes de saúde da família passa a ser um marco na atenção primária, uma vez que aproxima os profissionais dos usuários da área adscrita. Com foco no atendimento às famílias e passando a ser a equipe de referência de todos os moradores da comunidade, as equipes atuam como porta de entrada do sistema de saúde.

Tentando facilitar a articulação entre as especificidades das equipes de saúde da família e a necessidade de atenção à saúde mental, o Apoio Matricial surge como um processo de construção compartilhada, numa nova proposta de intervenção pedagógico-terapêutica ${ }^{12}$. Esses novos arranjos devem ser transversais, no sentido de produzir e estimular padrões de relação que perpassem todos trabalhadores e usuários, favorecendo a troca de informações e a ampliação do compromisso dos profissionais com a produção de saúde ${ }^{13}$.

O matriciamento favorece a corresponsabilização pelo usuário, por meio da interlocução entre a saúde mental e as equipes de ESF, atuando como uma retaguarda especializada de assistência, evitando, dessa forma, os encaminhamentos desnecessários a outros níveis de atendimento e aumentando a capacidade resolutiva de problemas de saúde pela equipe de referência ${ }^{12}$.

\section{METODOLOGIA}

Este estudo foi realizado por meio de uma Revisão Integrativa ${ }^{14}$, que é um método utilizado para agrupar os resultados de pesquisas primárias, sobre o mesmo assunto, com o objetivo de sintetizar e analisar esses dados para desenvolver uma explicação mais abrangente de um fenômeno específico.

São seis as etapas de elaboração da Revisão Integrativa: 1. Identificação do tema e seleção da hipótese ou questão de pesquisa para a elaboração da Revisão; 2. Estabelecimento de critérios para inclusão e exclusão dos artigos; 3. Definição das informações a serem extraídas dos estudos selecionados, com categorização dos mesmos; 4. Avaliação dos artigos incluídos na Revisão; 5. Interpretação dos resultados; 6. Apresentação da Revisão. Tais etapas são apresentadas ao longo do texto.

1. Identificação do tema e seleção da questão de pesquisa: que fatores têm sido identificados como facilitadores e como dificultadores da interlocução entre Atenção Primária à Saúde e Saúde Mental, propiciada pelo matriciamento em saúde mental?

2. Estabelecimento de critérios para inclusão e exclusão dos artigos: como critérios de inclusão, foram considerados artigos na íntegra, publicados em português, inglês e espanhol, que tratassem da temática pesquisada, contivessem as palavras-chaves (atenção primária, saúde mental e apoio matricial) e fossem publicados nos últimos 14 anos (1999 a 2013).

Os critérios de exclusão foram: artigos incompletos e apenas resumos, trabalhos não disponíveis online e que não tratassem da temática proposta. 
A coleta de dados ocorreu de junho de 2012 a janeiro de 2013. Foram acessados artigos publicados desde 1999, ano da formulação da concepção de apoio matricial por Gastão Wagner Campos, até 2013, indexados na base PubMed, no serviço da Biblioteca Nacional de Medicina Americana (NLM) e na Biblioteca Virtual em Saúde (BVS), que dá acesso às bases de dados: Literatura LatinoAmericana e do Caribe em Ciências da Saúde (LILACS), Medical Literature Analysis and Retrieval System Online (MEDLINE) e Índice Bibliográfico Espanhol em Ciências da Saúde (IBECS).
Para a busca dos artigos, foram utilizadas as supracitadas palavras-chave e suas combinações, nas línguas portuguesa, espanhola e inglesa.

3. Definição das informações a serem extraídas dos estudos selecionados, com categorização dos mesmos: as informações extraídas dos estudos selecionados foram buscadas nos itens título; ano de publicação; resumo; palavras-chave; objetivos; metodologia; e conclusões.

4. Avaliação dos artigos incluídos na Revisão: as informações pesquisadas nos artigos incluídos na Revisão foram compiladas no Quadro 1.

Quadro 1. Relação das publicações que compuseram a amostra do estudo de acordo com código, título e ano de publicação

\begin{tabular}{|c|c|c|c|c|}
\hline $\begin{array}{l}\text { Código } \\
\text { do estudo }\end{array}$ & Título & Ano & Revista & Autores \\
\hline A1 & $\begin{array}{l}\text { Avaliação das estratégias inovadoras na organização da } \\
\text { Atenção Primária à Saúde }\end{array}$ & 2012 & Revista de Saúde Pública & Campos et al. ${ }^{15}$ \\
\hline A 2 & $\begin{array}{l}\text { Onde está a criança?. Desafios e obstáculos ao apoio } \\
\text { matricial de crianças com problemas de saúde mental }\end{array}$ & 2012 & Physis & $\begin{array}{l}\text { Cavalcante, Jorge e } \\
\text { Santos }^{16}\end{array}$ \\
\hline A3 & $\begin{array}{l}\text { Apoio Matricial em saúde mental: alcances e limites na } \\
\text { atenção básica }\end{array}$ & 2012 & Saúde e Sociedade & Morais e Tanaka ${ }^{17}$ \\
\hline A 4 & $\begin{array}{l}\text { Perfil dos usuários de serviços de saúde mental do } \\
\text { município de Lorena/São Paulo }\end{array}$ & 2012 & Acta Paulista de Enfermagem & Pereira et al. ${ }^{18}$ \\
\hline A5 & $\begin{array}{l}\text { Apoio matricial como dispositivo do cuidado em } \\
\text { saúde mental na atenção primária: olhares múltiplos e } \\
\text { dispositivos para resolubilidade }\end{array}$ & 2012 & Ciência e Saúde Coletiva & Pinto et al. ${ }^{19}$ \\
\hline A6 & $\begin{array}{l}\text { Estratégia de apoio em saúde mental aos agentes } \\
\text { comunitários de saúde de Salvador/BA }\end{array}$ & 2012 & Saúde e Sociedade & Silva, Santos e Souza ${ }^{20}$ \\
\hline A7 & $\begin{array}{l}\text { Articulação entre serviços públicos de saúde nos cuidados } \\
\text { voltados à saúde mental infatojuvenil }\end{array}$ & 2012 & Cadernos de Saúde Pública & Delfini e Reis ${ }^{21}$ \\
\hline A8 & $\begin{array}{l}\text { Saúde mental na atenção primária à saúde: estudo } \\
\text { avaliativo em uma grande cidade brasileira }\end{array}$ & 2011 & Ciência e Saúde Coletiva & Campos et al. ${ }^{22}$ \\
\hline A9 & Apoio matricial e atenção primária em saúde & 2011 & Saúde e Sociedade & Cunha e Campos ${ }^{8}$ \\
\hline A10 & $\begin{array}{l}\text { Desafios do cuidado em saúde mental na estratégia saúde } \\
\text { da família }\end{array}$ & 2011 & $\begin{array}{l}\text { Revista Brasileira de Promoção } \\
\text { à Saúde }\end{array}$ & Cavalcante et al. ${ }^{23}$ \\
\hline A11 & $\begin{array}{l}\text { Centro de atenção psicossocial: convergência entre saúde } \\
\text { mental e coletiva }\end{array}$ & 2011 & Psicologia e Saúde & Ballarin et al. ${ }^{24}$ \\
\hline A12 & $\begin{array}{l}\text { Tecendo a rede assistencial em saúde mental com a } \\
\text { ferramenta matricial }\end{array}$ & 2011 & Physis & Sousa et al. ${ }^{25}$ \\
\hline A13 & $\begin{array}{l}\text { Avaliação das ações em saúde mental na estratégia de } \\
\text { saúde da família: necessidades e potencialidades }\end{array}$ & 2011 & $\begin{array}{l}\text { Revista Gaúcha de } \\
\text { Enfermagem }\end{array}$ & Cossetin e Olschowsky ${ }^{7}$ \\
\hline A14 & $\begin{array}{l}\text { Apoio matricial: um caminho de fortalecimento das redes } \\
\text { de atenção à saúde em Palmas - TO }\end{array}$ & 2011 & $\begin{array}{l}\text { Revista Brasileira de Ciências } \\
\text { e Saúde }\end{array}$ & Prestes et al. ${ }^{26}$ \\
\hline A15 & $\begin{array}{l}\text { Saúde mental na Estratégia Saúde da Família: a avaliação } \\
\text { de apoio matricial }\end{array}$ & 2010 & $\begin{array}{l}\text { Revista Brasileira de } \\
\text { Enfermagem }\end{array}$ & Mielke e Olchowsky ${ }^{27}$ \\
\hline A16 & $\begin{array}{l}\text { Implantação do matriciamento nos serviços de saúde de } \\
\text { Capivari }\end{array}$ & 2009 & Saúde e Sociedade & Aroma $^{28}$ \\
\hline A17 & $\begin{array}{l}\text { Saúde mental na atenção básica à saúde de Campinas, SP: } \\
\text { uma rede ou um emaranhado? }\end{array}$ & 2009 & Ciência e Saúde Coletiva & Figueiredo e Campos ${ }^{29}$ \\
\hline A18 & $\begin{array}{l}\text { O Apoio Matricial em Unidades de Saúde da Família: } \\
\text { experimentando inovações em saúde mental }\end{array}$ & 2009 & Saúde e Sociedade & Dimenstein et al. ${ }^{6}$ \\
\hline A19 & $\begin{array}{l}\text { O apoio matricial na perspectiva de coordenadoras de } \\
\text { equipes de saúde da família }\end{array}$ & 2009 & $\begin{array}{l}\text { Pesquisas e Práticas } \\
\text { Psicossociais }\end{array}$ & $\begin{array}{l}\text { Dimenstein, Galvão e } \\
\text { Severo }^{30}\end{array}$ \\
\hline A 20 & $\begin{array}{l}\text { Saúde mental e atenção primária: uma experiência com } \\
\text { agentes comunitários de saúde em Salvador-BA }\end{array}$ & 2009 & $\begin{array}{l}\text { Revista Brasileira de Promoção } \\
\text { à Saúde }\end{array}$ & Carneiro et al. ${ }^{31}$ \\
\hline A 21 & $\begin{array}{l}\text { Os CAPS e o trabalho em rede: tecendo o apoio matricial } \\
\text { na atenção básica }\end{array}$ & 2008 & Psicologia: Ciência e Profissão & $\begin{array}{l}\text { Bezerra e } \\
\text { Dimenstein }^{33}\end{array}$ \\
\hline
\end{tabular}




\section{RESULTADOS}

Os tópicos de Resultados e Discussão correspondem às etapas de Interpretação dos resultados (etapa 5) e Apresentação da Revisão (etapa 6), da Revisão Integrativa de Cooper. Utilizando-se os passos metodológicos descritos, foram identificadas 445 publicações nas diferentes bases de dados, das quais 50 foram excluídas por repetição, 3 por serem artigos publicados em idiomas não incluídos no estudo, 12 tinham textos incompletos ou não disponíveis e 358 foram rejeitadas após primeira análise de leitura do título e resumo por não incluírem atenção primária, saúde mental e apoio matricial. Dos 25 artigos restantes, 3 foram rejeitados por leitura dinâmica e procura das palavras-chave no texto (segunda análise) e 1 foi excluído por leitura integral do texto (terceira análise).

Em síntese, foram incluídos nesta revisão 21 artigos, em português, dos quais 14 foram publicados no ano de 2011 e 7, em 2012; dentre os trabalhos, 18 são pesquisas, 2 são artigos de reflexão e 1 é relato de experiência. Para coleta e posterior análise de dados, foi elaborado um instrumento especificando informações relativas a título, autoria e ano da publicação, tipo metodológico e objetivo do estudo.
Dos 21 textos selecionados, 5 traziam apenas aspectos positivos da implementação do matriciamento; 6 mostravam apenas resultados que impediam a concretização do apoio matricial, servindo de obstáculo para sua implantação; 10 artigos abordavam tanto aspectos positivos quanto negativos. Tais aspectos são apresentados nos Gráficos 1 e 2 .

Os aspectos positivos que aparecem com maior frequência nos artigos analisados são construção coletiva de saberes e melhor comunicação entre trabalhadores, usuários e gestores, através da implementação da rede de saúde (nove textos); melhor e maior conhecimento a respeito do tema Saúde Mental, através de encontros e trocas cotidianas entre os profissionais (sete textos); e, por fim, aumento na corresponsabilização, através do compartilhamento do cuidado, gerando um encaminhamento implicado (cinco textos).

Entre os aspectos negativos levantados após análise dos artigos, destacam-se a falta de uma rede de saúde, que proporcione maior diálogo entre os atores envolvidos no processo de cuidado (oito textos); dificuldade dos trabalhadores em compreender a real utilização do matriciamento, criando obstáculos para sua implantação (seis textos); e capacitação

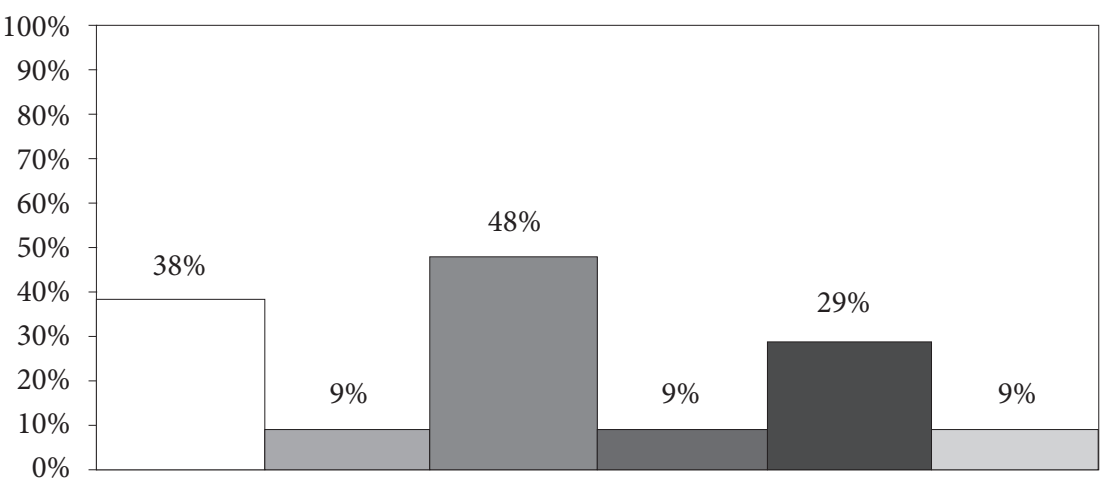

Melhor compreensão sobre Saúde Mental

Superação das práticas de medicalização

Construção da rede de saúde

Valorização do papel do Agente Comunitário

Emcaminhamento implicado

Implatação do NASF

NASF: Núcleo de Apoio à Saúde da Família

Gráfico 1. Aspectos positivos da implantação do matriciamento

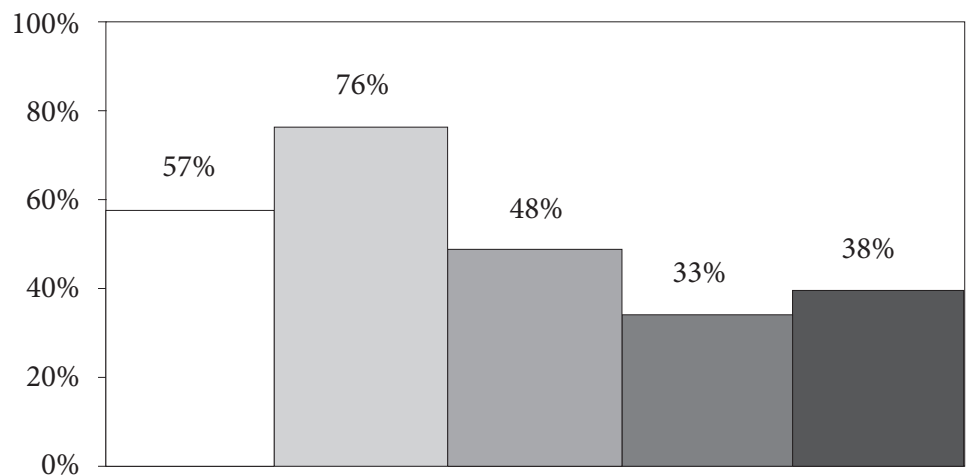

Gráfico 2. Aspectos negativos no processo de implantação do Matriciamento
Dificuldade de Entendimento / Implantação do Matriciamento

Falta de Rede de Saúde

Capacitação Precária dos Profissionais

Desresponsabilização Sobre o Usuário

Problemas de Gestão de Saúde Pública 
precária ou, por vezes, inexistente dos profissionais, tanto da Atenção Básica, quanto da Saúde Mental (cinco textos).

A análise dos dados foi realizada mediante leitura analítica, com a qual se pretendeu levantar aspectos relacionados à temática, organizá-los, interpretá-los, desenvolvê-los e refletir sobre eles. A pesquisa não apresentou a necessidade de aprovação pelo Comitê de Ética em Pesquisa, uma vez que manipula dados de livre acesso, não se tratando, portanto, de documentos que requeiram sigilo ético. As demais questões éticas foram preservadas, na medida em que todos os autores consultados foram devidamente referenciados no texto.

\section{DISCUSSÃO}

A análise dos artigos permitiu a identificação de duas categorias, percebidas com base no objetivo deste estudo, qual seja, caracterizar a utilização do apoio matricial como instrumento de articulação entre a Atenção Básica e a Saúde Mental. O critério de categorização de dados escolhido foi o semântico; logo, as fontes que apresentaram o mesmo contexto ou ideia foram agrupadas na redação das categorias.

As categorias levantadas após a análise foram abordadas em dois tipos: 1) aspectos que dificultam o matriciamento; 2) aspectos potencializadores do apoio matricial. O Quadro 2 apresenta a frequência com que esses aspectos aparecem nos estudos pesquisados.

\section{Aspectos que dificultam o matriciamento}

Falar em Saúde Mental sempre foi motivo de angústia para muitos profissionais de saúde. Isso se deve, em parte, à história de maus-tratos, isolamento, incompreensão do louco em nossa sociedade e, principalmente, ao preconceito em relação à loucura e à dificuldade dos trabalhadores em entrar em contato com o sofrimento do outro ${ }^{5,29}$. Além disso, a grande maioria das instituições de ensino superior, embora apresente o tema Saúde Mental em sua grade curricular, não prepara o profissional de uma maneira mais efetiva para o cuidado aos pacientes em sofrimento psíquico ou psiquiátrico, tampouco contribuem para tornar essa área mais atrativa ${ }^{17}$.

Quadro 2. Relação das categorias, com seus respectivos estudos codificados, e a porcentagem dos estudos presentes em cada categoria

\begin{tabular}{|c|c|c|}
\hline Categorias & Código dos estudos & $\%$ \\
\hline $\begin{array}{l}\text { Aspectos que } \\
\text { dificultam o } \\
\text { matriciamento }\end{array}$ & $\begin{array}{c}\mathrm{A} 3, \mathrm{~A} 4, \mathrm{~A} 5, \mathrm{~A} 6, \mathrm{~A} 7, \mathrm{~A} 8, \mathrm{~A} 9, \mathrm{~A} 10, \\
\mathrm{~A} 12, \mathrm{~A} 13, \mathrm{~A} 15, \mathrm{~A} 16, \mathrm{~A} 17, \mathrm{~A} 19, \\
\mathrm{~A} 20, \mathrm{~A} 21\end{array}$ & 76,2 \\
\hline $\begin{array}{l}\text { Aspectos } \\
\text { potencializadores do } \\
\text { apoio matricial }\end{array}$ & $\begin{array}{c}\mathrm{A} 1, \mathrm{~A} 2, \mathrm{~A} 3, \mathrm{~A} 7, \mathrm{~A} 9, \mathrm{~A} 10, \mathrm{~A} 11 \\
\mathrm{~A} 12, \mathrm{~A} 13, \mathrm{~A} 14, \mathrm{~A} 15, \mathrm{~A} 17, \mathrm{~A} 18 \\
\mathrm{~A} 19, \mathrm{~A} 21\end{array}$ & 71,4 \\
\hline
\end{tabular}

Porém, a partir da Reforma Psiquiátrica, com a desinstitucionalização do sujeito em sofrimento psíquico e a busca de novas práticas em saúde mental, não manicomiais, percebe-se um maior estímulo do cuidado desses indivíduos no contexto da atenção básica, já que esta, por meio da Estratégia de Saúde da Família, tem percebido um aumento na demanda de ações de Saúde Mental. A realidade dessas equipes demonstra que, cotidianamente, elas se deparam com problemas de "Saúde Mental", pois $56 \%$ das equipes de saúde da família avaliadas pelo Ministério da Saúde referem realizar "alguma ação de saúde mental"5.

Essa maior proximidade, no entanto, não diminui a sensação de apreensão do trabalhador que acolherá o usuário portador de transtornos mentais, pois os profissionais, na sua grande maioria, não se sentem preparados para tanto. Percebe-se que existe ainda uma carência de capacitação para os aspectos subjetivos do cuidar em saúde mental, como o manejo de crises, o relacionamento interpessoal e o instrumental terapêutico, que são apontados como requisitos fundamentais para atuar em Saúde Mental na Atenção Primária ${ }^{17}$. A falta de recursos de pessoal e de capacitação acabam por prejudicar o desenvolvimento de uma ação integral pelas equipes ${ }^{5}$.

Além disso, os trabalhadores de serviços da atenção básica e de serviços especializados em Saúde Mental apresentam dificuldade em compreender o que é o matriciamento. Percebe-se que não há clareza acerca da proposta de apoio matricial, muito menos há espaços para debates sobre matriciamento, bem como sobre outras estratégias possíveis para tecer uma rede de atenção capaz de fazer a articulação e a interligação entre os serviços integrantes da Saúde Mental e os demais no território ${ }^{25,29,30}$. Esses profissionais percebem a necessidade de apoio e instrumentalização sobre o tema para, dessa forma, fortalecer o apoio matricial, melhorando a integração com a rede de atenção em saúde d,8,23 $^{7}$.

Há também uma lógica na qual o profissional da atenção básica encaminha o usuário para serviços especializados, transferindo a responsabilidade do cuidado e dificultando a construção de ações conjuntas. A Saúde Mental, por sua vez, não cumpre seu papel de retaguarda assistencial especializada, não se apropriando dos casos e devolvendo o usuário à equipe de referência sem contato prévio, muitas vezes, sem preencher sequer o documento de contrarreferência, deixando evidente a falta de comunicação entre os níveis e dentro das próprias equipes ${ }^{8,15,23,26}$.

Vale lembrar que, infelizmente, existe uma cultura predominante que toma o saber profissional nuclear como propriedade privada, fortalecendo a prática de desresponsabilização do cuidado ao usuário, dificultando cada vez mais as ações em rede ${ }^{8}$. Fica clara a fragilidade, a fragmentação ou a inexistência de uma rede de cuidados e a falta de comunicação entre as equipes de saúde, o que inviabiliza, muitas vezes, o apoio matricial ${ }^{7,8}$. 
Enfim, tais aspectos dificultadores, como falta de capacitação profissional, incompreensão sobre o tema e inexistência da rede de cuidados, entre outros, acabam por tornarem-se empecilhos para o desenvolvimento de um trabalho conjunto, interdisciplinar, com constante troca de saberes, como o preconizado pelo matriciamento ${ }^{8,26,30,32}$.

\section{Aspectos potencializadores do matriciamento}

Apesar de os obstáculos supracitados interferirem profundamente na implantação do apoio matricial, dificultando, assim, sua função de interlocutor entre a Saúde Mental e a atenção primária, há muitos aspectos positivos que o confirmam como potente instrumento de cuidado colaborativo entre os profissionais que compõem uma rede de saúde.

$\mathrm{O}$ apoio matricial tem, como um de seus pontos fortes, o potencial de ser um dispositivo articulador da rede, não apenas promovendo a corresponsabilização nos diversos níveis de organização, mas também apoiando as equipes de Estratégia de Saúde da Família nas demandas cotidianas, além de contribuir na construção de ações de Saúde Mental no território ${ }^{27,30}$. Facilita, ainda, a elaboração de novos processos de trabalho, com enfoque multidisciplinar, possibilitando espaços de troca de experiências, conhecimentos, discussões e encontros que viabilizam a formação de projetos terapêuticos singularizados e de cuidado criativo. Associado a isso, somam-se a incorporação da Saúde Mental no fazer das equipes e a diversificação dos dispositivos de cuidado, que implicam em ações que impulsionam o fazer em conjunto e formas dialógicas de organização do trabalho ${ }^{29,30}$.

O apoio matricial propicia também a construção coletiva de saberes em Saúde Mental, garantindo espaço de cuidado aos Agentes Comunitários de Saúde, fomentando a implantação de redes do cotidiano, possibilitando, dessa forma, aos serviços e aos profissionais, uma maior aceitação e compreensão da Saúde Mental na atenção primária ${ }^{17,20,31}$. Além disso, o trabalho em conjunto ajuda os profissionais a se sentirem mais seguros e a perceberem que não estão sozinhos para resolver os casos, que podem compartilhar responsabilidades e se fortalecerem para lidar melhor com situações do dia a dia ${ }^{21}$. Assim, fica evidente a ênfase do matriciamento como um suporte especializado de saberes e ações para trabalhadores, surgindo como importante forma de capacitação para os profissionais, contribuindo para o aumento da capacidade resolutiva das equipes ${ }^{7}$.

A partir do aumento da resolutividade, conseguida por meio do apoio matricial, ocorre, por consequência, a diminuição das interconsultas e, principalmente, os encaminhamentos desnecessários ao especialista ${ }^{28}$. Assim, o apoio matricial se mostra como um potente instrumento de definição de fluxos, qualificação das equipes e promoção de uma assistência conjunta e compartilhada ${ }^{8,19,21,22}$.

\section{CONCLUSÃO}

Após a análise e a discussão dos textos, fica evidente que o matriciamento, arranjo organizacional que visa outorgar suporte técnico em áreas específicas às equipes responsáveis pelo desenvolvimento de ações de saúde para a população por meio do compartilhamento e corresponsabilização de casos $^{5}$, embora com alguns entraves que dificultam sua implantação -, mostra-se como uma importante ferramenta de interlocução entre a Saúde Mental e a atenção básica.

Dentre os principais fatores levantados que dificultam sua função de articulador entre a Saúde Mental e a atenção primária podem estar a fragilidade da rede de saúde, que compromete, assim, o diálogo entre os atores envolvidos no processo de cuidado; a dificuldade dos trabalhadores em entender com clareza os princípios e os objetivos do matriciamento; a capacitação precária ou, por vezes, inexistente dos profissionais da saúde; a desresponsabilização com o usuário, contrária ao que é preconizado pelo apoio matricial, por meio do encaminhamento indiscriminado ao especialista, sem preocupação com a longitudinalidade do cuidado. Merecem destaque também os problemas relativos à gestão de Saúde Pública, pois, inúmeras vezes, "a política de Saúde Mental não prevê apoio matricial em seus eixos de direcionamento", além de apresentar "escassez de recursos humanos e de serviços, rigidez nas agendas e cobrança por produtividade que atrapalham, ou até mesmo, inviabilizam o funcionamento do apoio matricial" ${ }^{7,21}$.

Já entre os aspectos potencializadores que se sobressaem na análise dos textos, estão: a construção coletiva de saberes e a melhor comunicação entre trabalhadores, usuários e gestores, através da implementação da rede de saúde, como o preconizado pelo apoio matricial; uma maior difusão do tema da Saúde Mental, através de encontros e trocas cotidianas entre os profissionais, desmistificando-o e tornando-o mais próximo dos profissionais que compõem a rede de cuidados; o aumento na corresponsabilização, por meio do compartilhamento do cuidado, estabelecendo-se um encaminhamento implicado e fortalecimento do vínculo; pode-se também citar a superação das práticas de medicalização, puramente curativas, individuais e hospitalocêntricas; a valorização do papel do Agente Comunitário de Saúde, que passa a ser peça-chave na comunicação entre as equipes e os usuários; e, por fim, a implantação do Núcleo de Apoio à Saúde da Família (NASF), criado pelo Ministério da Saúde por meio da portaria GM $\mathrm{n}^{\circ} 154 / 2008^{33}$, que se tornou uma das formas mais eficazes, atualmente, para se consolidar o apoio matricial.

O NASF deve ser constituído por equipes multiprofissionais para atuarem conjuntamente com as Equipes de Saúde da Família, criando espaços de discussões e trocas constantes, tendo como eixos principais a responsabilização, a gestão 
compartilhada e o apoio à coordenação do cuidado. Apesar de existir desde 2008, ainda são poucos os municípios que contam com essa retaguarda especializada.

Cabe, portanto, aos gestores a tarefa de implantar o NASF e, aos profissionais e à comunidade, cobrar desses gestores a funcionalidade desse dispositivo que surge para fortalecer o matriciamento. Cabe também aos trabalhadores e aos usuários exigirem que o apoio matricial funcione como articulador entre a Saúde Mental e a atenção primária, propiciando, assim, o favorecimento de trocas de saberes, de compartilhamento no cuidado, de corresponsabilização e, principalmente, de uma atenção mais humanizada.

Além disso, a partir do momento em que todos os trabalhadores da saúde passarem a utilizar o apoio matricial efetivamente como uma nova maneira de produzir saúde de forma compartilhada, permanecendo a responsabilização pelo usuário com a atenção primária, contribuiremos para reforçar a importância da Estratégia de Saúde da Família como organizadora da atenção primária e principal porta de entrada do sistema.

Espera-se que esta revisão tenha provocado a reflexão acerca do apoio matricial na articulação da Saúde Mental no contexto da atenção primária à saúde, revelando, assim, seus aspectos, sobretudo, facilitadores do trabalho em rede; porém, muitas vezes, esse apoio é negligenciado ou desvalorizado pelos gestores e trabalhadores de saúde, mas pode proporcionar a todos os atores envolvidos no cuidado um atendimento mais humanizado, pautado no respeito e na valorização dos sujeitos.

Assim, percebe-se a necessidade da implantação de medidas que consolidem o apoio matricial como uma estratégia de qualificação do cuidado em saúde que atenda às necessidades dos sujeitos e que esteja assegurada enquanto uma política de saúde. Com este estudo, buscou-se dar maior visibilidade aos princípios que cercam o apoio matricial no contexto da Saúde Mental, mostrando seus limites e possibilidades e promovendo a divulgação de experiências exitosas neste campo.

\section{REFERÊNCIAS}

1. Brasil. Ministério da Saúde. Portaria MS/GM No 2.488, de 21 de Outubro de 2011. Brasília: Diário Oficial da União. Seção 1, p. 48-55, out. 2011.

2. Nunes ED. Saúde coletiva: uma história recente de um passado remoto. In: Campos GWS, Minayo MCS, Akerman M, Junior MD, Carvalho YM (orgs.). Tratado de Saúde Coletiva. São Paulo: Hucitec, 2009. p. 19-39.

3. Costa FB, Trindade MAN, Pereira MLT. A inserção do biomédico no programa de saúde da família. Revista Eletrônica Novo Enfoque. 2010;11(11):27-33

4. Campinas. Secretaria de Saúde. Texto base para discussão sobre matriciamento. Prefeitura Municipal de Campinas. Set. 2006.

5. Brasil. Ministério da Saúde. Saúde mental e atenção básica: o vínculo e o diálogo necessários - inclusão das ações de saúde mental na atenção básica, $\mathrm{n}^{\circ}$ 01. Brasília: Secretaria de Atenção à Saúde; Departamento de Ações Programáticas Estratégicas; Departamento de Atenção Básica; Coordenação Geral de Saúde Mental e Coordenação de Gestão da Atenção Básica; 2003.

6. Dimenstein M, Severo AK, Brito M, Pimenta AL, Medeiros V, Bezerra E. O Apoio Matricial em Unidades de Saúde da Família: experimentando inovações em saúde mental. Saúde Soc. 2009;18(1):63-74.

7. Cossetin A, Olschowsky A. Avaliação das ações em saúde mental na estratégia de saúde da família: necessidades e potencialidades. Rev Gaúch Enferm. 2011;32(3):495-501.

8. Cunha GT, Campos GWS. Apoio matricial e atenção primária em saúde. Saúde Soc. 2011;20(4):961-70.

9. Brasil. Ministério da Saúde. Secretaria de Atenção à Saúde. DAPE. Coordenação Geral de Saúde Mental. Reforma psiquiátrica e política de saúde mental no Brasil. Documento apresentado à Conferência Regional de Reforma dos Serviços de Saúde Mental: 15 anos depois de Caracas. OPAS. Brasília, novembro de 2005.
10. Brasil. Ministério da Saúde. Centro de Atenção Psicossocial - CAPS [Internet]. Brasília;2012. [cited 2012 Dec20]. Available from:http://portal. saude.gov.br/portal/saude/visualizar_texto.cfm?idtxt=29797\&janela=

11. Brasil. Departamento de Atenção Básica. Histórico de Cobertura da Saúde da Família [Internet]. Brasília; 2012. [cited 2013 Jan 4]. Available from: http://dab.saude.gov.br/portaldab/historico_cobertura_sf.php

12. Brasil. Ministério da Saúde. Guia prático de matriciamento em saúde mental. Brasília: Centro de Estudo e Pesquisa em Saúde Coletiva; 2011.

13. Brasil. Ministério da Saúde. HumanizaSUS: equipe de referência e apoio matricial. Brasília: Secretaria-executiva. Núcleo Técnico da Política Nacional de Humanização; 2004.

14. Cooper HM. Scientific guidelines for conducting integrative research reviews. Rev Educ Res. 1982;52(2):291-302.

15. Campos RTO, Campos GWS, Ferrer AL, Corrêa CRS, Madureira PR, Gama CAP, et al. Avaliação das estratégias inovadoras na organização da Atenção Primária à Saúde. Rev Saúde Pública. 2012;46(1):43-50.

16. Cavalcante CM, Jorge MSB, Santos DCM. Onde está a criança?. Desafios e obstáculos ao apoio matricial de crianças com problemas de saúde mental. Physis. 2012;22(1):161-78.

17. Morais APP, Tanaka OY. Apoio Matricial em saúde mental: alcances e limites na atenção básica. Saúde Soc. 2012;21(1):161-70.

18. Pereira MO, Souza JM, Costa AM, Vargas D, Oliveira MAF, Moura WN Perfil dos usuários de serviços de saúde mental do município de Lorena/ São Paulo. Acta Paul Enferm. 2012;25(1):48-54.

19. Pinto AGA, Jorge MSB, Vasconcelos MGF, Sampaio JJC, Lima GP, Bastos VC, et al. Apoio matricial como dispositivo do cuidado em saúde mental na atenção primária: olhares múltiplos e dispositivos para resolubilidade. Ciênc Saúde Coletiva. 2012;17(3):653-60 
20. Silva CB, Santos JE, Souza RC. Estratégia de apoio em saúde mental aos agentes comunitários de saúde de Salvador/BA. Saúde Soc. 2012;21(1):153-60.

21. Delfini PSS, Reis AOA. Articulação entre serviços públicos de saúde nos cuidados voltados à saúde mental infatojuvenil. Cad. Saúde Pública. 2012;28(2):357-66

22. Campos RO, Gama CA, Ferrer AL, Santos DVD, Stefanello S, Trapé TL, et al. Saúde mental na atenção primária à saúde: estudo avaliativo em uma grande cidade brasileira. Ciênc Saúde Coletiva. 2011;16(12):4643-52.

23. Cavalcante CM, Pinto DM, Carvalho AZT, Jorge MSB, Freitas CHA. Desafios do cuidado em saúde mental na estratégia saúde da família. Rev Bras Promoç Saúde. 2011;24(2):102-8.

24. Ballarin MLGS, Carvalho FB, Ferigato SH, Miranda IMS, Magaldi CC. Centro de atenção psicossocial: convergência entre saúde mental e coletiva. Psicol Estud. 2011;16(4):603-11.

25. Sousa FSP, Jorge MSB, Vasconcelos MGF, Barros MMM, Quinderé PHD, Gondim LGF. Tecendo a rede assistencial em saúde mental com a ferramenta matricial. Physis. 2011;21(4):1579-99.

26. Prestes LIN, Araújo AC, Costa CS, Nascimento MDW, Oliveira DA. Apoio matricial: um caminho de fortalecimento das redes de atenção à saúde em Palmas - TO. Rev Bras Ciênc Saúde. 2011;15(2):215-8.
27. Mielke FB, Olchowsky A. Saúde mental na Estratégia Saúde da Família: a avaliação de apoio matricial. Rev Bras Enferm. 2010;63(6):900-7.

28. Aroma EC. Implantação do matriciamento nos serviços de saúde de Capivari. Saúde Soc. 2009;18(Supl. 1):26-36.

29. Figueiredo MD, Campos RO. Saúde mental na atenção básica à saúde de Campinas, SP: uma rede ou um emaranhado? Ciênc Saúde Coletiva. 2009;14(1):129-38.

30. Dimenstein $\mathrm{M}$, Galvão VM, Severo $\mathrm{AK}$. O apoio matricial na perspectiva de coordenadoras de equipes de saúde da família. Pesquisa e Práticas Psicossoais. 2009;4(1):37-48.

31. Carneiro AC, Oliveira ACM, Santos MMS, Alves MS, Casais NA, Santos JE. Saúde mental e atenção primária: uma experiência com agentes comunitários de saúde em Salvador-BA. Revista Bras Promoç Saúde. 2009;22(4):264-71.

32. Bezerra E, Dimenstein M. Os CAPS e o trabalho em rede: tecendo o apoio matricial na atenção básica. Psicol Ciênc Prof. 2008;28(3):632-45.

33. Brasil. Ministério da Saúde. Diretrizes do NASF: Núcleo de Apoio à Saúde da Família. Série A. Normas e Manuais Técnicos. Cadernos de Atenção Básica, n² 27. Brasília: Secretaria de Atenção à Saúde; Departamento de Atenção Básica; 2009.

Recebido em: 01/04/2013

Aprovado em: 11/06/2013 